\title{
Bounding tree-width via contraction on the projective plane and torus
}

\author{
Evan Morgan \\ Wakely Consulting Group \\ 9777 Pyramid Ct., Suite 260 \\ Englewood, CO 80112, USA \\ morganevan@gmail.com
}

\author{
Bogdan Oporowski \\ Department of Mathematics \\ Louisiana State University \\ Baton Rouge, LA 70803, USA \\ bogdan@math.1su.edu
}

Submitted: Jul 9, 2012; Accepted: Sep 26, 2015; Published: Oct 16, 2015

Mathematics Subject Classifications: 05C10, 05C15, 05C83

\begin{abstract}
If $X$ is a collection of edges in a graph $G$, let $G / X$ denote the contraction of $X$. Following a question of Oxley and a conjecture of Oporowski, we prove that every projective-planar graph $G$ admits an edge-partition $\{X, Y\}$ such that $G / X$ and $G / Y$ have tree-width at most three. We prove that every toroidal graph $G$ admits an edge-partition $\{X, Y\}$ such that $G / X$ and $G / Y$ have tree-width at most three and four, respectively.
\end{abstract}

\section{Introduction}

In 2005, Gonçalves settled an old conjecture of Chartrand, Geller, and Hedetniemi [2] by proving the following $[6]$ :

Theorem 1 (Gonçalves). Every planar graph admits an edge-partition into two outerplanar graphs.

Before that result appeared, Ding, Oporowski, Sanders, and Vertigan [5] (and, independently, Kedlaya [7]) proved the following partial result:

Theorem 2 (Ding, Oporowski, Sanders, and Vertigan; Kedlaya). Every planar graph admits an edge-partition into two series-parallel graphs.

We can rephrase Theorem 2 as follows:

Theorem 3. Every planar graph $G$ admits an edge-partition $\left\{E_{1}, E_{2}\right\}$ such that $G \backslash E_{1}$ and $G \backslash E_{2}$ are series-parallel. 
If $G$ is a connected graph embedded on a surface, and $G^{*}$ is a surface dual of $G$, then the reader can notice that the deletion of an edge of $G$ corresponds to the contraction of the corresponding edge of $G^{*}$; this phenomenon is investigated more fully in Section 2 . Deletion and contraction may, in this way, be viewed as dual operations. Furthermore, the reader can notice that $K_{4}$, embedded on the plane, is isomorphic to its own dual. Therefore it is an easy exercise to prove that if $G$ is a plane graph and $G^{*}$ is a surface dual of $G$, then $K_{4}$ is a minor of $G$ if and only if $K_{4}$ is a minor of $G^{*}$. This implies the following corollary of Theorem 3 .

Corollary 4. Every plane graph $G$ admits an edge-partition $\left\{E_{1}, E_{2}\right\}$ such that $G / E_{1}$ and $G / E_{2}$ are series-parallel.

With this in mind, Oxley [9] asked the following question.

Question 5 (Oxley). For a cographic matroid $M$, is there a bipartition $\{S, T\}$ of the ground set such that $M \backslash S$ and $M \backslash T$ are series-parallel?

Oxley's question led the second author to conjecture the following generalization of Corollary 4.

Conjecture 6 (Oporowski, unpublished). Every graph $G$ admits a partition $\left\{E_{1}, E_{2}\right\}$ of its edges such that each of $G / E_{1}$ and $G / E_{2}$ is series-parallel.

As a first observation, we note that contracting edges in a graph does not raise its tree-width. In particular, the tree-width of a graph is equal to the maximum tree-width of its 2-connected blocks. We investigate this issue more fully in Section 2, and prove that it suffices to consider only 2-connected graphs.

As a second observation, we note that contracting a spanning tree in a (connected) graph results in a graph with a single vertex. Therefore, if a graph $G=(V, E)$ contains two edge-disjoint spanning trees $T_{1}$ and $T_{2}$, we can partition the edge set into $E_{1}=E\left(T_{1}\right)$ and $E_{2}=E \backslash E\left(T_{1}\right)$; in this case, $G / E_{1}$ and $G / E_{2}$ are singletons. Both Nash-Williams [8] and Tutte [12] proved theorems characterizing the graphs that contain $k$ edge-disjoint spanning trees. A nice statement and proof can be found on pages 48-52 of [4].

Theorem 7 (Nash-Williams; Tutte). A graph contains $k$ edge-disjoint spanning trees if and only if for every partition $\left(V_{1}, \ldots, V_{l}\right)$ of its vertex set, it has at least $k(l-1)$ distinct $\left(V_{i}, V_{j}\right)$-edges, where $i$ and $j$ are in $\{1, \ldots, l\}$.

The following immediate corollary is relevant to our purposes.

Corollary 8. Every 4-connected graph contains two edge-disjoint spanning trees.

Therefore Conjecture 6 holds for all 4-connected graphs. The question remains: What about graphs of connectivity two and three?

Demaine, Hajiaghayi, and Mohar [3] have investigated the problem of partitioning the edge set of a graph embedded on a surface, such that contracting any partite set bounds the tree-width. They proved the following very powerful theorem. 
Theorem 9 (Demaine, Hajiaghayi, and Mohar). For every two integers $g$ and $k$ such that $g \geqslant 0$ and $k \geqslant 2$, and for every graph $G$ of Euler genus at most $g$, the edges of $G$ can be partitioned into $k$ sets such that contracting any one of the sets results in a graph of tree width at most $O\left(g^{2} k\right)$.

In this paper, we examine a few specific surfaces - namely the plane, the projective plane, and the torus - to improve significantly the bounds obtained in [3]. We prove the following two theorems.

Theorem 10. Every projective-planar graph $G$ admits a bipartition $\{X, Y\}$ of $E(G)$ such that $G / X$ and $G / Y$ have tree-width at most three.

Theorem 11. Every toroidal graph $G$ admits a bipartition $\{X, Y\}$ of $E(G)$ such that $G / X$ and $G / Y$ have tree-width at most three and four, respectively.

Theorem 10 is restated and proved in Section 3 as Theorem 22. Theorem 11 is restated and proved in Section 5 as Theorem 31. Conjecture 6 in general remains unresolved, but Theorems 10 and 11 may be considered partial results.

In Section 2, we provide a variety of technical lemmas used in the proof of Theorem 22 . Our method overall, in Sections 2 and 3, is roughly as follows:

1. Reduce the problem to the case of cubic, 2-connected graphs;

2. Look at a surface dual $G^{*}$ of an arbitrary cubic, 2-connected projective plane graph $G$;

3. Find a disk in the projective plane that contains all the vertices of $G^{*}$ and that induces a connected (spanning) subgraph;

4. Decompose $G^{*}$ into nested subgraphs called distance layers;

5. Obtain a bipartition of $E\left(G^{*}\right)$ by grouping edges in alternating distance layers;

6. Prove that the corresponding bipartition $\{X, Y\}$ of $E(G)$ satisfies the conclusion of Theorem 22.

In Section 4, we prove some technical lemmas used in the proof of Theorem 31. Our method, overall, in Sections 4 and 5, is roughly as follows:

1. Reduce the problem to cubic, 2-connected toroidal graphs;

2. Prove that certain 4-connected plane triangulations admit edge-partitions into two outerplanar graphs;

3. Use (2) to prove that all planar graphs admit a particular edge-partition into two series-parallel graphs;

4. Find a suitable set of pairwise non-adjacent edges in our toroidal graph whose deletion produces a planar graph; 
5. Use the partition from (3) on the planar graph from (4) to produce an edge-partition $\{X, Y\}$ of our toroidal graph $G$;

6. Prove that $\{X, Y\}$ satisfies the conclusion of Theorem 31 .

Any terminology not defined here can be found in [4]. In this paper, we allow graphs to have loops and multiple edges. Let $G$ be a graph. A block of $G$ is a subgraph of $G$ isomorphic to a loop, a cut-edge, or a maximal 2-connected subgraph of $G$. A graph $H$ is a minor of a $G$ if $H$ can be obtained from a subgraph of $G$ by contracting edges. If $T$ is a tree and $\mathcal{V}=\left\{V_{t}\right\}_{t \in V(T)}$ is a collection of sets of vertices of $G$, then the pair $(T, \mathcal{V})$ is a tree-decomposition of $G$ if the following hold:

(T1) $V(G)=\bigcup_{t \in V(T)} V_{t}$;

(T2) For every edge $e \in E(G)$, there is a vertex $t \in V(T)$, such that $e \in G\left[V_{t}\right]$;

(T3) If $t_{1}, t_{2}, t_{3}$ are vertices of $T$ so that $t_{2}$ lies on the unique $\left(t_{1}, t_{3}\right)$-path in $T$, then $V_{t_{1}} \cap V_{t_{3}} \subseteq V_{t_{2}}$.

The sets $V_{t}$ are called bags. Given a tree-decomposition of $G$, the width of the decomposition is one less than the size of a largest bag. The tree-width of $G$, notated as $\operatorname{tw}(G)$, is the minimum width among all tree-decompositions of $G$. Note that the tree-width of a tree with at least one edge is one. It is well-known that a graph has tree-width at most two if and only if it has no $K_{4}$-minor; we call such graphs series-parallel.

In this paper, we freely consider graphs as topological spaces, in the natural way, and we therefore mention the following notation: For a subset $S$ of a topological space, the closure of $S$ is denoted $\bar{S}$, the boundary of $S$ is denoted $\partial S$, and the interior of $S$ is denoted $\stackrel{\circ}{S}$. The model we use for the projective plane is based on the unit disk in $\mathbb{R}^{2}$ with the supremum metric $d_{\infty}$, defined as $d_{\infty}\left(\left(x_{1}, y_{1}\right),\left(x_{2}, y_{2}\right)\right)=\max \left(\left|x_{2}-x_{1}\right|,\left|y_{2}-y_{1}\right|\right)$. Then, the projective space is understood as the unit disk $U$, which appears as a square $\{(x, y):-1 \leqslant x \leqslant 1,-1 \leqslant y \leqslant 1\}$, in which every point $(x, y)$ in its boundary $\partial U$ is identified with the antipodal point $(-x,-y)$. We will consider piecewise-linear embeddings of graphs in the projective plane, in which each vertex is represented as a point in the interior of $U$, and each edge is either represented by a single polygonal arc that avoids $\partial U$, or by two disjoint polygonal arcs, each meeting $\partial U$ in exactly one point, with these two points being antipodal; the edges of the latter type will also be called antipodal. It is well known that any graph embedding in the projective plane may be expressed in such a way without changing its combinatorial structure. An open disk $D$ in $U$ is clean with respect to a graph $G$ embedded in the projective plane if it is concentric with $U$ and has radius sufficiently close to 1 (again, measured by $d_{\infty}$ ) so that it contains all vertices of $G$, the boundary of $D$ meets only the antipodal edges of $G$, and each antipodal edge appears in $U \backslash D$ as two disjoint straight line segments.

If a graph $G$ is embedded in a surface $\Gamma$, a face of $G$ is a connected component of $\Gamma \backslash G$. An edge $e$ is incident to a face $F$ if $e \subseteq \partial F$. The representativity of an embedded graph $G$ is the least number of points at which a non-contractible cycle meets $G$, and is denoted $\operatorname{rep}(G)$. 
If $H$ is a subgraph of $G$, an $H$-bridge of $G$ is a subgraph of $G$ consisting either of

(1) an edge whose endpoints are in $H$ but is itself not in $H$, or

(2) a connected component $B$ of $G \backslash H$, together with the edges with one endpoint in $B$ and one endpoint in $H$.

The vertices of attachment of an $H$-bridge $B$ of $G$ are the vertices of $B$ that lie in $H$.

\section{The case of the projective plane - introductory results}

Arnborg, Corneil, and Proskurowski [1] proved the following forbidden-minor characterization of graphs with tree-width at most three.

Theorem 12 (Arnborg, Corneil, and Proskurowski). A graph $G$ has tree-width at most three if and only if none of $K_{5}, M_{6}, M_{8}, M_{10}$ is a minor of $G$; where $M_{6}$ is the octahedron, $M_{8}$ is the Möbius ladder on eight vertices (also called the Wagner graph), and $M_{10}$ is the pentagonal prism, as depicted in Figure 1.

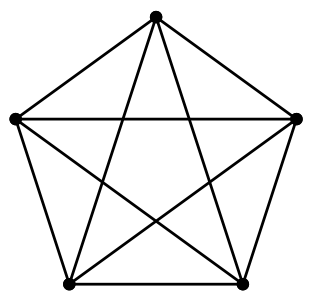

$K_{5}$

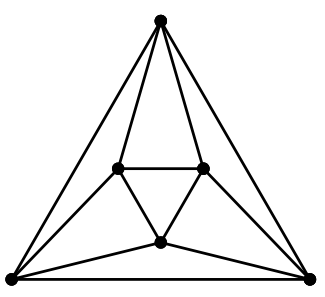

$M_{6}$

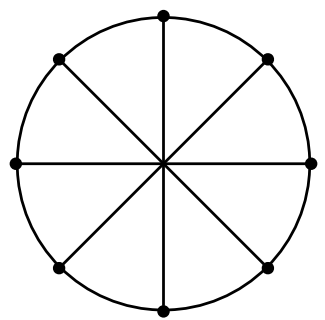

$M_{8}$

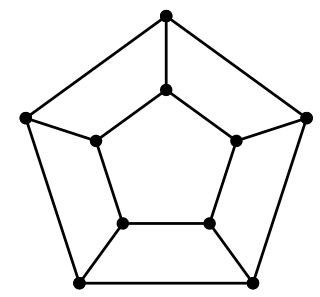

$M_{10}$

Figure 1: The forbidden minors for graphs with tree-width at most three.

The next three lemmas express basic facts about tree-width and contraction, and together they imply that it suffices to prove Theorems 22 and 31 for cubic, 2-connected graphs. We omit their proofs.

Lemma 13. If $G$ is a graph and $H$ is a 2-connected minor of $G$, then $H$ is a minor of some 2-connected block of $G$.

Lemma 14. Let $G$ be a connected graph, let $G_{1}, G_{2}, \ldots, G_{k}$ be the blocks of $G$, and let $m \geqslant 1$ and $n \geqslant 1$. Then if each $E\left(G_{i}\right)$, for $i \in\{1, \ldots, k\}$, admits a bipartition $\left\{X_{i}, Y_{i}\right\}$ such that $\operatorname{tw}\left(G_{i} / X_{i}\right) \leqslant m$ and $\operatorname{tw}\left(G_{i} / Y_{i}\right) \leqslant n$, then $E(G)$ admits a bipartition $\{X, Y\}$ such that $\operatorname{tw}(G / X) \leqslant m$ and $\operatorname{tw}(G / Y) \leqslant n$.

Lemma 15. Let $G$ and $G^{\prime}$ be graphs, such that $G^{\prime}$ is cubic and can be obtained from $G$ by suppressing vertices of degree two and one, and by repeatedly splitting vertices of degree greater than three. If $E\left(G^{\prime}\right)$ admits a partition $\left\{X^{\prime}, Y^{\prime}\right\}$ such that $\operatorname{tw}\left(G^{\prime} / X^{\prime}\right) \leqslant m$ and $\operatorname{tw}\left(G^{\prime} / Y^{\prime}\right) \leqslant n$, where $m \geqslant 2$ and $n \geqslant 2$, then $E(G)$ admits a partition $\{X, Y\}$ such that $\operatorname{tw}(G / X) \leqslant m$ and $\operatorname{tw}(G / Y) \leqslant n$. 
The following lemma finds an appropriate "outer layer" with controlled structure, whose deletion yields a connected planar graph.

Lemma 16. Let $G$ be a 2-connected, non-planar, projective plane triangulation. There is a closed curve $\alpha$ in the projective plane that bounds a disk D such that the following hold:

(1) $\alpha \cap V(G)=\varnothing$;

(2) $V(G) \subsetneq D$;

(3) $|\alpha \cap e| \in\{0,2\}$ for every $e \in E(G)$;

(4) The graph induced by the edges that avoid $\alpha$ is connected.

Proof. Let $G$ be a 2-connected embedding of a non-planar graph in the projective plane in which each face has exactly three edges in its boundary. We can represent the projective plane by a unit disk $U$ as measured by $d_{\infty}$, and can also represent $G$ as a piecewise-linear drawing in $U$. Let $D$ be a clean disk with respect to this drawing, let $\alpha$ be the boundary of $D$, and let $H$ be the graph induced by the edges that are contained in $D$. Assume now that the representation of $G$ was initially chosen so that $H$ has the smallest number of connected components possible. Notice that if $H$ is connected, then the conditions (1)-(4) in the statement of the lemma are satisfied. See Figure 2.

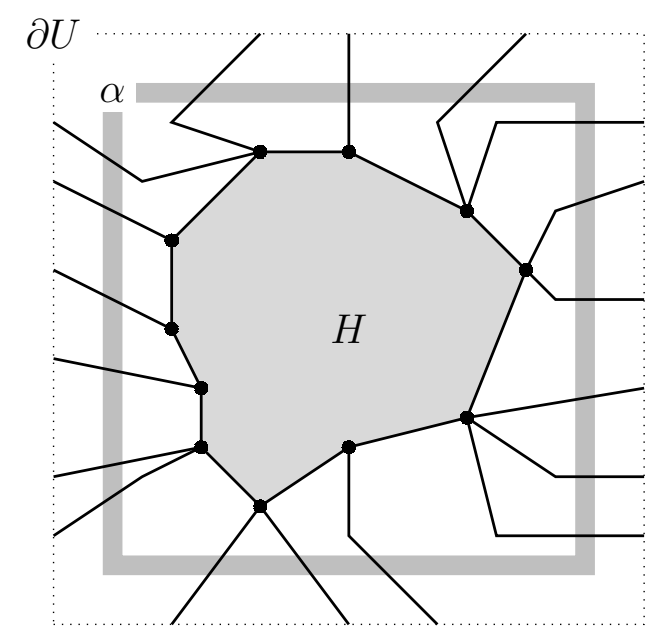

Figure 2: When $H$ is connected, the conclusion follows.

Suppose now that $H$ has $k$ connected components, with $k \geqslant 2$. Let $b_{1}, \ldots, b_{p}$ be the points in $\alpha \cap G$. For each $b_{i}$, with $i \in\{1, \ldots, p\}$, there is an edge-segment, lying in $D$, with endpoints $b_{i}$ and $v_{i}$, for some vertex $v_{i}$ of $G$. And we know that each vertex of $G$ lies in some component of $H$; we say that $b_{i}$ is connected to the said component of $H$. Notice that $b_{1}, \ldots, b_{p}$ divides $\alpha$ up into $p$ closed segments, which we call $S_{1}, \ldots, S_{p}$. For each $S_{m}$ whose endpoints $b_{i}$ and $b_{j}$ are connected to distinct components of $H$, pick a point in $S_{m}$ 
that avoids $\left\{b_{1}, \ldots, b_{p}\right\}$; let the resulting points be $z_{1}, \ldots, z_{q}$. Notice that $z_{1}, \ldots, z_{q}$ divide $\alpha$ up into $q$ closed segments, which we call $T_{1}, \ldots, T_{q}$. Then the points in $\left\{b_{1}, \ldots, b_{p}\right\} \cap T_{i}$, for each $i \in\{1, \ldots, q\}$, are all connected to the same component of $H$. We say that a component of $H$ is represented by $T_{i}$ if the points of $\left\{b_{1}, \ldots, b_{p}\right\} \cap T_{i}$ are connected to that component. Since $G$ is connected, we know that every component of $H$ is represented by at least one of $T_{1}, \ldots, T_{q}$. See Figure 3 .

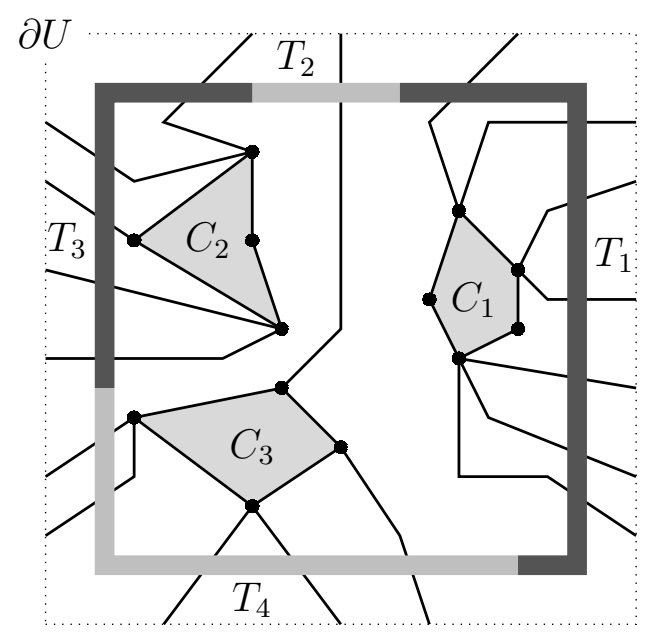

Figure 3: The components $C_{1}, C_{2}, C_{3}$ of $H$ are represented by the segments $T_{1}, T_{2}, T_{3}$, $T_{4}$.

Claim 17. Some component of $H$ is represented by exactly one of $T_{1}, \ldots, T_{q}$.

We will use induction to prove that if $C$ is a component of $H$ that is represented by more than one of $T_{1}, \ldots, T_{q}$, then there is a component $C^{\prime}$ of $H$ that is represented by fewer of $T_{1}, \ldots, T_{q}$ than is $C$.

If Claim 17 fails, then pick a component $C$ of $H$, segments $T_{i}$ and $T_{j}$, and a sequence of segments $T_{1}^{\prime}, \ldots, T_{r}^{\prime}$ such that the following hold:

(1) $T_{i}$ and $T_{j}$ are distinct representatives of $C$;

(2) None of $T_{2}^{\prime}, \ldots, T_{r-1}^{\prime}$ are representatives of $C$;

(3) $T_{1}^{\prime}=T_{i}$;

(4) $T_{r}^{\prime}=T_{j}$;

(5) $\left|T_{l}^{\prime} \cap T_{l+1}^{\prime}\right|=1$, for all $l \in\{1, \ldots, r-1\}$;

(6) no other choice of $C, T_{i}, T_{j}$, and $T_{1}^{\prime}, \ldots, T_{r}^{\prime}$ satisfying (1)-(5) yields a smaller value of $r$. 
(In Figure 3 , if we take $C$ to be $C_{3}$, then a suitable sequence would be $T_{2}, T_{3}, T_{4}$.) Let $C^{\prime}$ be a component of $H$ represented by at least one segment in $T_{2}^{\prime}, \ldots, T_{r-1}^{\prime}$. According to the projective plane topology, we know that all the segments that represent $C^{\prime}$ lie in $T_{2}^{\prime}$, $\ldots, T_{r-1}^{\prime}$, and (6) ensures that the component $C^{\prime}$ is innermost with respect to $T_{1}^{\prime}, \ldots, T_{r}^{\prime}$. Therefore $C^{\prime}$ is represented by fewer of $T_{1}, \ldots, T_{q}$ than is $C$, and hence Claim 17 holds.

We will now prove the following statement, which contradicts our choice of the representation of $G$, and therefore concludes the proof of the lemma.

Claim 18. There is a disk $D^{\prime}$ and a closed curve $\alpha^{\prime}$ bounding $D^{\prime}$ that satisfy conditions (1), (2), and (3) of the statement of the lemma (when $\alpha^{\prime}$ replaces $\alpha$, and $D^{\prime}$ replaces $D$ ), such that the graph induced by the edges contained in $D^{\prime}$ has fewer than $k$ components.

Various parts of the construction of $\alpha^{\prime}$ are illustrated in Figure 4. Let $C$ be a component of $H$ that is represented by exactly one of $T_{1}, \ldots, T_{q}$. Without loss of generality, suppose $C$ is represented by $T_{1}$.

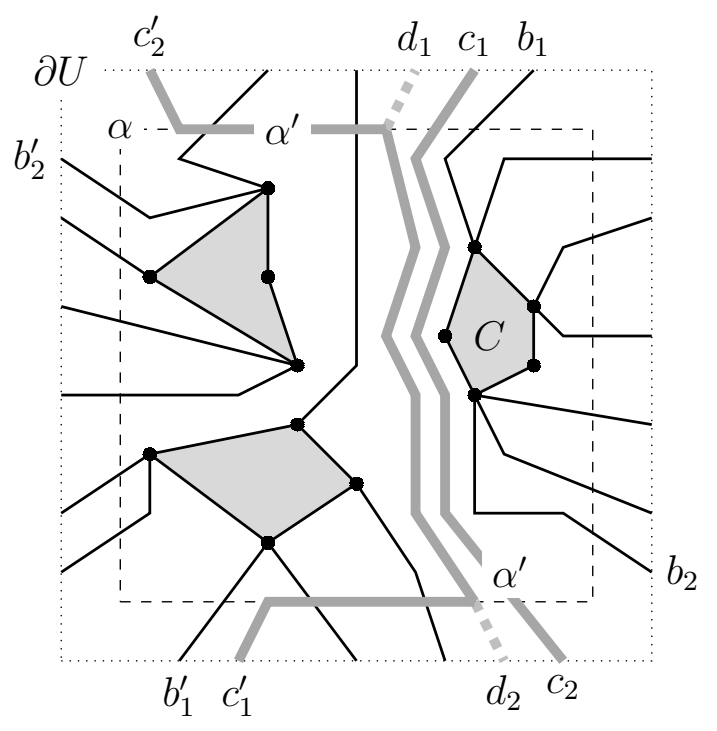

Figure 4: We aim to re-route $\alpha$ from Figure 3 to obtain the curve $\alpha^{\prime}$ shown here.

Let $S_{1}, \ldots, S_{t}$ be the components of $G \backslash D$ whose closures meet $T_{1}$, listed in order in which they meet $T_{1}$. Let $b_{1}=S_{1} \cap \partial U$, let $b_{2}=S_{t} \cap \partial U$, and let $\beta$ be a polygonal arc that is a part of $\partial U$, has $b_{1}$ and $b_{2}$ as endpoints, and whose closure meets all of $S_{1}, \ldots, S_{t}$. Let $b_{1}^{\prime}$ and $b_{2}^{\prime}$ be the antipodal points of, respectively, $b_{1}$ and $b_{2}$. Note that if one of $b_{1}^{\prime}$ and $b_{2}^{\prime}$ lies on $\beta$, then so does the other, and then $H$ has only one other component besides $C$, for which the analogous statement does not hold. So, we may assume that neither $b_{1}^{\prime}$ nor $b_{2}^{\prime}$ lies on $\beta$. Let $\beta^{\prime}=\partial U \backslash \beta$.

Since $G$ is 2-connected and has a piecewise-linear embedding, $C$ contains a path whose union with $S_{1}$ and $S_{t}$ forms a polygonal arc $\beta^{\prime \prime}$ such that the closed disk bounded by $\beta \cup \beta^{\prime \prime}$ contains $C \cup S_{1} \cup \cdots \cup S_{t}$. It is easy to see that since the embedding of $G$ is clean, $U$ contains a polygonal arc $\gamma$, sufficiently close to $\beta^{\prime \prime}$, that avoids $G$, meets $U \backslash D$ in two 
disjoint straight line segments, and meets $\partial U$ exactly at its endpoints $c_{1}$ and $c_{2}$, which lie on $\beta^{\prime}$. Let $c_{1}^{\prime}$ and $c_{2}^{\prime}$ be the points antipodal to, respectively, $c_{1}$ and $c_{2}$. By switching the names of $c_{1}$ and $c_{2}$, if necessary, we may assume that the points $b_{1}, c_{1}, c_{2}^{\prime}, b_{2}^{\prime}, b_{1}^{\prime}, c_{1}^{\prime}$, $c_{2}$, and $b_{2}$ appear on $\partial U$ in the order listed. Further, $U$ contains another polygonal arc $\delta$, sufficiently close to $\gamma$, that avoids both $G$ and $\gamma$, meets $U \backslash D$ in two disjoint line segments, one of which meets $\partial U$ in a point $d_{1}$ that lies on $\beta^{\prime}$ between $c_{1}$ and $c_{2}^{\prime}$, while the other meets $\partial U$ in a point $d_{2}$ that lies on $\beta^{\prime}$ between $c_{1}^{\prime}$ and $c_{2}$. Let the points in which $\delta$ meets $\alpha$ be $d_{1}^{\prime \prime}$ and $d_{2}^{\prime \prime}$ so that $d_{1}, d_{2}^{\prime \prime}, d_{1}^{\prime \prime}$, and $d_{2}$ appear on $\delta$ in the order listed. Let $\varepsilon_{1}$ be a straight line segment that joins $c_{1}^{\prime}$ to a point $c_{1}^{\prime \prime}$ on $\alpha$ so that $\varepsilon_{1}$ avoids all of $G, \gamma$, and $\delta$, and, similarly, let $\varepsilon_{2}$ be a straight line segment that joins $c_{2}^{\prime}$ to a point $c_{2}^{\prime \prime}$ on $\alpha$ so that $\varepsilon_{2}$ avoids all of $G, \gamma, \delta$, and $\varepsilon_{1}$. Let $\zeta_{1}$ and $\zeta_{2}$ be segments of $\alpha$ that avoid $\gamma$, and join, respectively, $c_{1}^{\prime \prime}$ to $d_{1}^{\prime \prime}$, and $c_{2}^{\prime \prime}$ to $d_{2}^{\prime \prime}$.

Now we define $\alpha^{\prime}=\gamma \cup\left(\delta \cap D_{i}\right) \cup \varepsilon_{1} \cup \varepsilon_{2} \cup \zeta_{1} \cup \zeta_{2}$. Then $\alpha^{\prime}$ and the disk $D^{\prime}$ it bounds satisfy the assertion 18 , and the conclusion of the lemma follows.

Duality in topological graph theory is a far less versatile concept than, for example, in matroid theory, since all duals are tied to a particular embedding. And any particular graph may admit numerous distinct embeddings, with variety in the number of faces. If there are embeddings of a graph $G$ with distinct numbers of faces, then any surface duals of these embeddings will not be isomorphic, since they will have a different number of vertices. For example, we may embed $K_{4}$ in the torus in the two ways represented in Figure 5 .
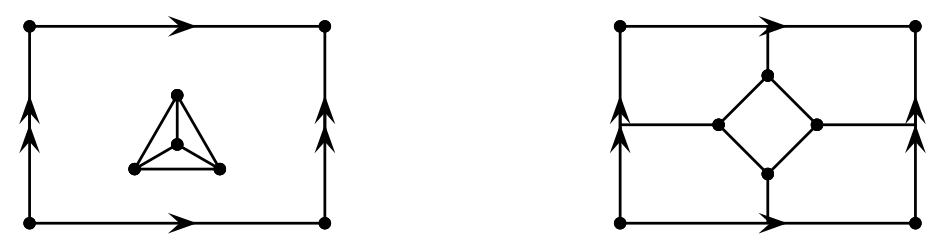

Figure 5: Toroidal embeddings of $K_{4}$, with a different number of faces.

In Figure 5, we see that the embedding on the left has four faces, and the embedding on the right has two faces. Therefore the surface duals of these embeddings will have distinct numbers of vertices. Hence the surface duals of these embeddings are non-isomorphic, as graphs.

Given a graph $G$ embedded in a surface, the following easy lemma, which we mention without proof, allows us to speak of the surface dual of $G$, which is a graph, not embedded in a surface, and is isomorphic to all surface duals of $G$.

Lemma 19. Let $G$ be a graph embedded in a surface. Then all surface duals of $G$ are isomorphic, as graphs.

The following lemma expresses an elementary fact about graph duality on the projective plane. 
Lemma 20. Let $G$ be a non-planar projective plane graph, and let $G^{*}$ be a surface dual of $G$. Let $B_{1}$ and $B_{2}$ be distinct blocks of $G$ such that $E\left(B_{1}\right) \cap \partial F$ and $E\left(B_{2}\right) \cap \partial F$ are non-empty, for some face $F$ of $G$. Let $E_{B_{1}}^{*}$ and $E_{B_{2}}^{*}$ be the edges of $G^{*}$ corresponding, via duality, to $E\left(B_{1}\right)$ and $E\left(B_{2}\right)$, respectively. Then $E_{B_{1}}^{*}$ and $E_{B_{2}}^{*}$ lie in distinct blocks of $G^{*}$.

Proof. Assuming the conditions of the lemma, we leave it to the reader to check that the vertex of $G^{*}$ corresponding to $F$ induces a 1-separation in $G^{*}$ that separates $B_{1}$ from $B_{2}$. (The non-planarity of $G$ ensures that precisely one block is non-planar. The other blocks are arranged accordingly.)

The next lemma is another elementary fact about graph duality. We omit its proof. It is the central premise of the proof-techniques in the main results of this chapter.

Lemma 21. Let $G$ be a connected graph embedded on the projective plane, and let $G^{*}$ be a surface dual of $G$. Let $X$ be a subset of edges of $G$, and let $X^{*}$ be the edges of $G^{*}$ corresponding, by duality, to the edges $X$. Then $G / X$ is isomorphic, as a graph, to the surface dual of $G^{*} \backslash X^{*}$.

\section{The case of the projective plane - main result}

We are now ready to prove our main result on projective-planar graphs.

Theorem 22. For any projective-planar graph $G$, there is a bipartition $\{X, Y\}$ of $E(G)$ such that $G / X$ and $G / Y$ have tree-width at most three.

Proof. Let $G$ be a projective-planar graph. If $G$ is planar, the conclusion follows from Corollary 4. Suppose, therefore, that $G$ is not planar. By Theorem 12, it suffices to find a bipartition $\{X, Y\}$ of $E(G)$ such that $G / X$ and $G / Y$ contain no minor isomorphic to $K_{5}, M_{6}, M_{8}$, and $M_{10}$.

By Lemmas 14 and 15, we may suppose that $G$ is 2-connected and cubic. Embed $G$ in the projective plane, and let $G^{*}$ be a surface dual of $G$. Then $G^{*}$ is a 2-connected, non-planar, projective plane triangulation. Lemma 16 gives us a particular closed curve $\alpha$ and disk $D$. Let $G_{-1}^{*}$ be the subgraph of $G^{*}$ induced by the edges that meet $\alpha$. Let $V_{0}$ consist of the vertices of $G^{*}$ that lie on the boundary of the face of $G^{*} \backslash E\left(G_{-1}^{*}\right)$ that contains $\alpha$. See Figure 6 . For $i \in\{1,2,3, \ldots\}$, let $V_{i}$ consist of the vertices of $G^{*}$ that are a distance $i$ from $V_{0}$. For $i \in\{0,1,2, \ldots\}$, let $G_{i}^{*}$ be the graph induced by the edges $e$ that satisfy one of the following:

(1) both endpoints of $e$ lie in $V_{i}$; or

(2) $e$ has one endpoint in $V_{i}$ and one endpoint in $V_{i+1}$.

We call the graphs $G_{i}^{*}$ distance layers. Let $X^{*}=\bigcup_{k \geqslant 0} E\left(G_{2 k-1}^{*}\right)$, and let $Y^{*}=$ $\bigcup_{k \geqslant 0} E\left(G_{2 k}^{*}\right)$. Let $X$ and $Y$ consist of the edges of $G$ that correspond, via duality, to the 
edges in $X^{*}$ and $Y^{*}$, respectively. Then $\{X, Y\}$ is a bipartition of the edges of $G$. We now prove that $G / X$ and $G / Y$ have no minors isomorphic to $K_{5}, M_{6}, M_{8}$, and $M_{10}$. By Lemma 21, we know that $G / X$ and $G / Y$ are isomorphic to the surface duals of $G^{*} \backslash X^{*}$ and $G^{*} \backslash Y^{*}$, respectively.
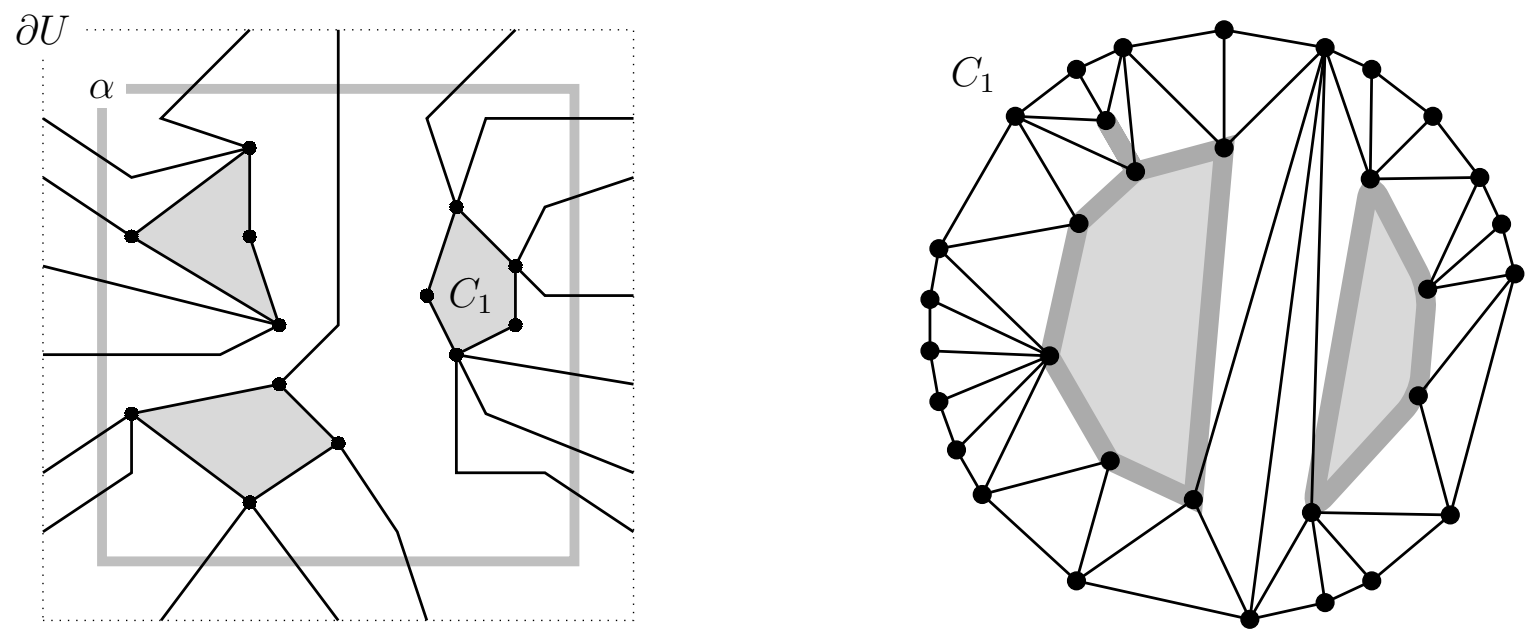

Figure 6: Left: The boundary of the shaded regions contains $V_{0}$. Note that the graph represented here, $G^{*}$, is a triangulation; for clarity, only some representative edges are shown. Right: A closer look at $C_{1}$. The shaded regions represent components of $G_{1}^{*} \cup$ $G_{2}^{*} \cup G_{3}^{*} \cup \cdots$ that lie in the disk bounded by $C_{1}$.

We now examine the structure of the surface duals of $G^{*} \backslash X^{*}$ and $G^{*} \backslash Y^{*}$. We know that $G^{*} \backslash X^{*}=G_{0}^{*} \cup G_{2}^{*} \cup G_{4}^{*} \cup \cdots$ and $G^{*} \backslash Y^{*}=G_{-1}^{*} \cup G_{1}^{*} \cup G_{3}^{*} \cup \cdots$. We know as well that $G_{i}^{*}$ and $G_{j}^{*}$ are vertex-disjoint if $|i-j|>1$. Then each connected component of $G^{*} \backslash X^{*}$ is a connected component of some $G_{2 i}^{*}$, with $i \geqslant 0$; and each connected component of $G^{*} \backslash Y^{*}$ is a connected component of some $G_{2 i-1}^{*}$, with $i \geqslant 0$.

By Lemma 20, we can conclude the following: No block $B$ of the surface dual of $G^{*} \backslash X^{*}$ (or $G^{*} \backslash Y^{*}$ ) contains edges corresponding, via duality, to two distinct blocks of $G^{*} \backslash X^{*}$ (or $\left.G^{*} \backslash Y^{*}\right)$.

Hence, using Lemma 13, it suffices to show that for each block $B$ of $G^{*} \backslash X^{*}$ and $G^{*} \backslash Y^{*}$, the surface dual of $B$ contains no minor isomorphic to $K_{5}, M_{6}, M_{8}$, and $M_{10}$. We now prove the following:

Claim 23. The surface duals of $G^{*} \backslash X^{*}$ and $G^{*} \backslash Y^{*}$ contain no $K_{5}$ - and no $M_{8}$-minor.

Since $E\left(G_{-1}^{*}\right)$ is in $X^{*}$, we know that $G^{*} \backslash X^{*}$ is embedded in the disk $D$. Therefore we can find a surface dual of $G^{*} \backslash X^{*}$ that lies in $D$; and since $D$ is a disk, this surface dual is planar. Therefore, since $K_{5}$ and $M_{8}$ are not planar, the surface dual of $G^{*} \backslash X^{*}$ contains no $K_{5^{-}}$and no $M_{8}$-minor.

Let $F_{-1}$ be the face of $G^{*} \backslash E\left(G_{0}^{*}\right)$ in which $\left(E\left(G_{0}^{*}\right)\right)^{\circ}$ lies. Then $F_{-1}$ is a face of $G^{*} \backslash Y^{*}$. Let $f_{-1}$ be the vertex of the surface dual of $G^{*} \backslash Y^{*}$ corresponding to $F_{-1}$, and suppose, without loss of generality, that $f_{-1} \in D$. 
We first prove that $G_{-1}^{*}$ has only one face. Suppose that two edges $u v$ and $u w$ in $G_{-1}^{*}$ are adjacent in the ordering induced by $\alpha$. Since $G^{*}$ is a triangulation, we know that $v w$ is an edge of $G_{0}^{*}$. Let $\alpha^{\prime}$ be a component of $\alpha \backslash G^{*}$ with endpoints $\alpha \cap u v$ and $\alpha \cap u w$. Then $\alpha^{\prime}$ lies in $F_{-1}$. Since $\alpha^{\prime}$ was chosen arbitrarily, we know that all components of $\alpha \backslash G^{*}$ lie in $F_{-1}$. Therefore $G_{-1}^{*}$ lies in $\overline{F_{-1}}$. Hence $G_{-1}^{*}$ has only one face. See Figure 7 . Hence the surface dual of $G_{-1}^{*}$ has only one vertex, namely $f_{-1}$, and $\left|E\left(G_{-1}^{*}\right)\right|$ loops.

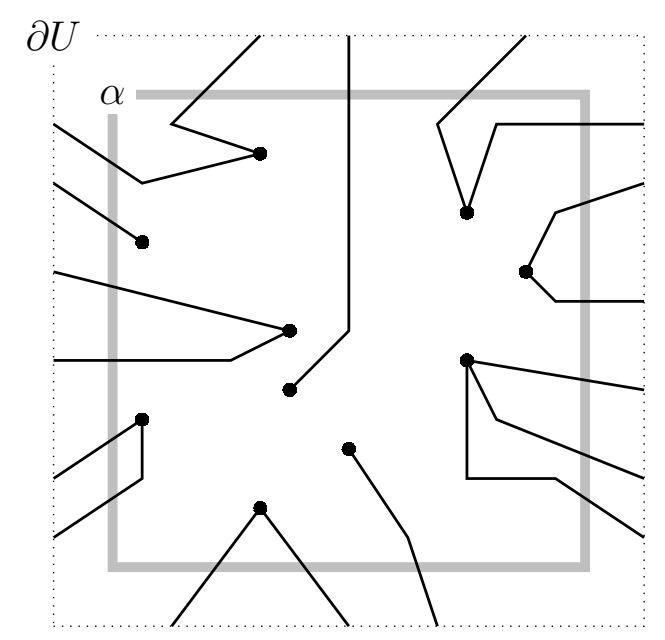

Figure 7: $G_{-1}^{*}$ has only one face.

Notice that $G^{*} \backslash\left(Y^{*} \cup G_{-1}^{*}\right)$ lies in $D$. Therefore we can find a surface dual of $G^{*} \backslash\left(Y^{*} \cup\right.$ $G_{-1}^{*}$ ) that lies in $D$, ensuring that the vertex corresponding to the face containing $F_{-1}$ is $f_{-1}$; and since $D$ is a disk, we know that this surface dual is planar. Therefore the surface dual of $G^{*} \backslash Y^{*}$ is a planar graph containing a vertex $f_{-1}$, to which we add $\left|E\left(G_{-1}^{*}\right)\right|$ loops at $f_{-1}$. Therefore the surface dual of $G^{*} \backslash Y^{*}$ contains no $K_{5^{-}}$and no $M_{8}$-minor. This concludes the proof of Claim 23.

The following definition will be useful: for each $i \in\{0,1,2, \ldots\}$, an internal face of $G_{i}^{*}$ is a face of $G_{i}^{*}$ that avoids $\alpha$. We now prove the following:

Claim 24. For each $i \in\{0,1,2, \ldots\}$, every $\left(V_{i}, V_{i}\right)$-edge $e$ that is incident to an internal face of $G_{i}^{*}$ lies in a 3 -cycle of $G_{i}^{*}$.

Let $i \in\{0,1,2, \ldots\}$ and let $u v$ be a $\left(V_{i}, V_{i}\right)$-edge that is incident to an internal face $F$ of $G_{i}^{*}$. Then there is a vertex $w$ and an edge $u w$, such that $w \neq v$ and $u w$ is incident to $F$. Since $G^{*}$ is a triangulation, we know that there is a triangular face $F^{\prime}$ of $G^{*}$ such that the following hold:

(1) $u v$ is incident to $F^{\prime}$;

(2) $F^{\prime} \subseteq F$. 
Let $w^{\prime}$ be the unique vertex incident to $F^{\prime}$ and distinct from $u$ and $v$. Since $w^{\prime}$ is adjacent to $u$, we know that $w^{\prime} \in V_{i} \cup V_{i+1}$. Therefore $u w^{\prime}$ and $v w^{\prime}$ are edges of $G_{i}^{*}$. Then $u v w^{\prime}$ is a 3-cycle of $G_{i}^{*}$ containing $u v$. This concludes the proof of Claim 24.

Using the definition of $G_{i}^{*}$, with $i \in\{0,1,2, \ldots\}$, and Claim 24, we see that each connected component of $G_{i}^{*}$, with $i \in\{0,1,2, \ldots\}$, is of the form described by the following construction:

(1) Let $T$ be a tree;

(2) For each $v \in V(T)$, let $P_{v}$ be a graph consisting of either an edge or a cycle with at least three edges, such that $P_{u}$ and $P_{v}$ are disjoint when $u \neq v$;

(3) For each edge $e$ in $T$, with endpoints $u$ and $v$, let $x_{e, u}$ and $x_{e, v}$ be arbitrary vertices of $P_{u}$ and $P_{v}$, respectively;

(4) Let $L^{\prime}$ be the graph formed from the graph $\bigcup_{v \in V(T)} P_{v}$ by identifying, for every $u v \in E(T)$, the vertices $x_{u}$ and $x_{v}$;

(5) Embed $L^{\prime}$ in $D$;

(6) Let $L$ be the graph (embedded in $D$ ) formed from $L^{\prime}$ by doing the following, for each cycle $C$ in $L_{0}$ :

(a) Let $D_{C}$ be the closed disk bounded by $C$;

(b) Let $Z_{C}$ be a finite collection of points in $D_{C}$;

(c) Embed any number of $(V(C), V(C))$-edges in $D_{C} \backslash Z_{C}$;

(d) Embed $\left(V(C), Z_{C}\right)$-edges in such a way that each $(V(C), V(C))$-edge lies in a 3-cycle.

See Figures 8 and 9 for illustrations of the construction.

We see that each block of $G_{i}^{*}$, with $i \in\{1,2, \ldots\}$, that contains more than one edge is contained in some component of $G_{0}^{*} \backslash\left\{E\left(P_{v}\right): v \in V(T)\right.$, and $P_{v}$ is an edge $\}$. Therefore, it suffices for us to prove the following:

Claim 25. The surface dual of an arbitrary component of $G_{0}^{*} \backslash\left\{E\left(P_{v}\right): v \in V(T)\right.$, and $P_{v}$ is an edge $\}$ contains no $M_{6}$ - and no $M_{10}$-minor.

Let $F_{0}$ be the face of $G_{0}^{*}$ that contains $\alpha$. We know that the boundary of $F_{0}$ may contain cycles of $G_{0}^{*}$; and we know that the blocks of $G_{0}^{*}$ (other than the blocks that consist of single edges) are those subgraphs of $G_{0}^{*}$ that are contained in the closed disks bounded by those cycles. Let $C$ be a cycle contained in the boundary of $F_{0}$. (Then $C=P_{v}$ for some $v \in V(T)$.) Let $D_{1}$ be the closed disk bounded by $C$. We consider now the edges of $G_{0}^{*}$ embedded in $D_{1}$. Let $J$ consist of all chords of $C$. (Note that all such chords are embedded in $D_{1}$.) Let $\left\{v_{1}, v_{2}, \ldots, v_{k}\right\}=V_{1} \cap D_{1}$; and for each $i \in\{1, \ldots, k\}$, let $S_{i}$ consist of all $\left(V_{0}, v_{i}\right)$-edges. Let $K=\left\{S_{1}, \ldots, S_{k}\right\}$. Then $K$ consists of all $\left(V_{0}, V_{1}\right)$-edges that lie in $D_{1}$. We know that every edge of $G_{0}^{*}$ embedded in $D_{1}$ is in $C \cup J \cup K$. Then 


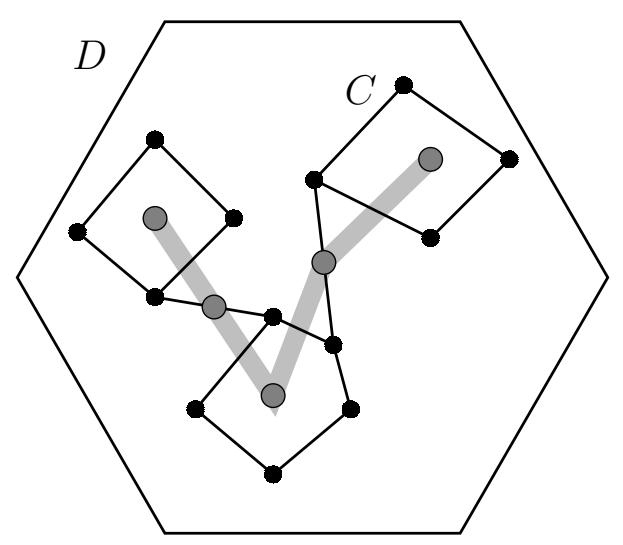

Figure 8: We see here the first five steps in the construction. The tree $T$ (here, a path) is represented with thick gray vertices and edges.
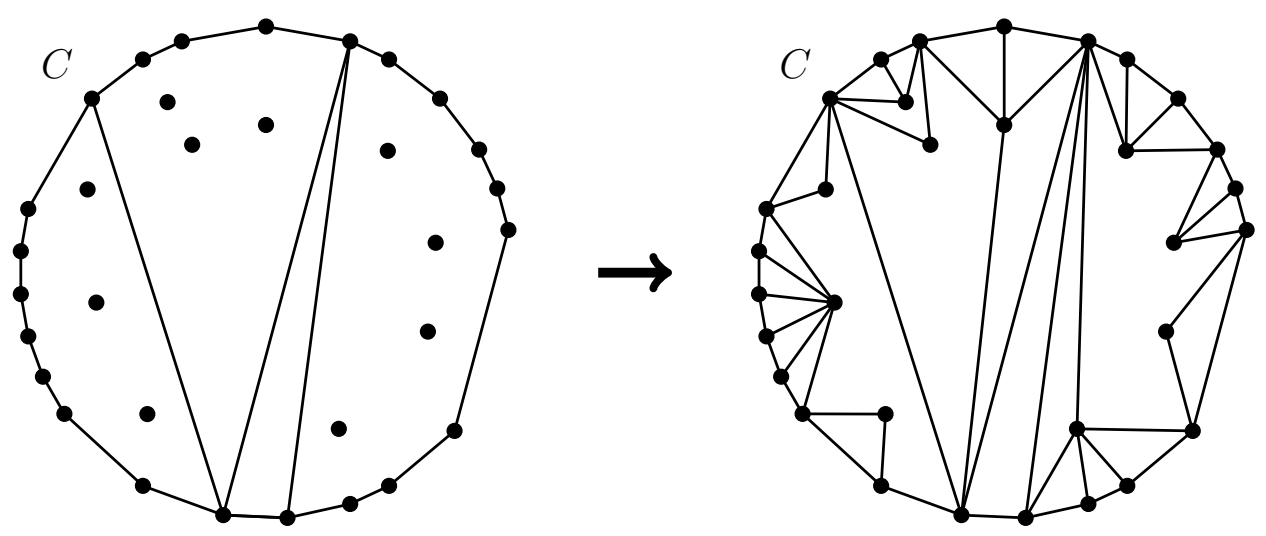

Figure 9: We see here step six of the construction. The figure on the left shows steps (6a), (6b), and (6c). The figure on the right shows step (6d).

$G^{*}[C \cup J \cup K]$ is an arbitrary component of $G_{0}^{*} \backslash\left\{E\left(P_{v}\right): v \in V(T)\right.$, and $P_{v}$ is an edge $\}$. We must therefore show that the surface dual of $G^{*}[C \cup J \cup K]$ contains no $M_{6^{-}}$and no $M_{10}$-minor.

We now construct a surface dual of $G^{*}[C \cup J \cup K]$. Let $f_{0}$ be a point in $D \cap D_{1}$. Notice that the surface dual of $G^{*}[C \cup J]$ is a tree, with all its leaves identified (notice that these leaves all lie outside of $D_{1}$ ); let $R_{0}$ be such a graph, embedded in $D_{1}$. Without loss of generality, we may assume the following:

(1) $f_{0}$ is the vertex of $R_{0}$ to which the leaves were identified;

(2) $R_{0}$ avoids $\left\{v_{1}, \ldots, v_{k}\right\}$.

Given $R_{0}$, we obtain a sequence $R_{1}, \ldots, R_{k}$ of graphs by performing the following inductive process on $R_{i-1}$ : 
(1) Let $F_{1}^{i}$ be the face of $G^{*}\left[C \cup J \cup\left\{S_{1} \cup \cdots \cup S_{i-1}\right\}\right]$ in which $v_{i}$ lies;

(2) Let $f_{i}$ be the vertex of $R_{i-1}$ corresponding to $F_{1}^{i}$;

(3) Let $\left(n_{1}, n_{2}, \ldots, n_{l}\right)$ be the rotation scheme at $f_{i}$ of $R_{i-1}$;

(4) Delete $f_{i}$ (and all the edges incident to it) from $R_{i-1}$ and embed a cycle $C_{i}$ of length $\left|S_{i}\right|$ (possibly a loop or doubled edge) in the resulting face such that $C_{i}$ bounds a closed disk that avoids $\left\{v_{i+1}, \ldots, v_{k}\right\} \cup\left(R_{i-1} \backslash f_{i}\right)$;

(5) For each $j \in\{1, \ldots, l\}$, embed the appropriate $\left(V\left(C_{i}\right), n_{j}\right)$-edge.

Thusly we obtain $R_{k}$, which is a surface dual of $G^{*}[C \cup J \cup K]$. If $M_{6}$ or $M_{10}$ is to be a minor of $R_{k}$, then $R_{k}$ must contain two disjoint, nested cycles $Z_{1}$ and $Z_{2}$, and three pairwise disjoint $\left(Z_{1}, Z_{2}\right)$-paths. See Figure 10.

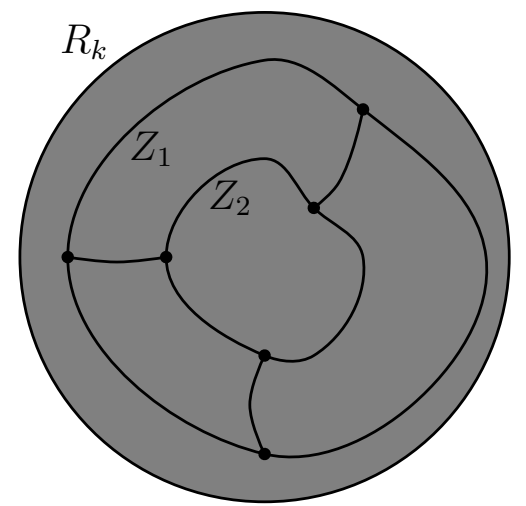

Figure 10: When $M_{6}$ or $M_{10}$ is a minor of $R_{k}$, then $R_{k}$ contains this substructure.

We prove now that $R_{k}$ contains no such cycles. Let $Z_{1}$ and $Z_{2}$ be disjoint nested cycles in $R_{k}$, and suppose that $Z_{2}$ is contained in the disk bounded by $Z_{1}$. Notice that cycles in $R_{k}$ are of two types: those that contain $f_{0}$ and those that do not contain $f_{0}$. In step (4) of the construction of $R_{i}$ from $R_{i-1}$, we specified that the closed disk bounded by $C_{i}$ avoid $\left\{v_{i+1}, \ldots, v_{k}\right\}$. Therefore no pair of $C_{1}, \ldots, C_{k}$ are nested. Since $\left\{v_{1}, \ldots, v_{k}\right\}$ are pairwise non-adjacent in $G_{0}^{*}$, we know that no two distinct $C_{i}$ and $C_{j}$ (with $i \neq j$ ) share an edge. Therefore, every cycle in $R_{k}$ that avoids $f_{0}$ is in $\left\{C_{1}, \ldots, C_{k}\right\}$. Therefore $Z_{2}=C_{i}$ for some $i \in\{1, \ldots, k\}$, and $Z_{1}$ contains $f_{0}$. Then $Z_{1} \backslash f_{0}$ is a path in the tree-like structure $R_{k} \backslash f_{0}$, and we know that there are not two disjoint $\left(Z_{2}, Z_{1} \backslash f_{0}\right)$-paths in $R_{k} \backslash f_{0}$. Therefore three pairwise disjoint $\left(Z_{1}, Z_{2}\right)$-paths do not exist in $R_{k}$. Hence $R_{k}$ contains no $M_{6^{-}}$and no $M_{10}$-minor. This concludes the proof of Claim 25 and Theorem 22.

\section{The case of the torus — introductory results}

In this section, we need a few specialized definitions. Suppose that $G$ is a plane graph with a 3 -cycle $x y z$. We define the interior of $x y z$, notated int $(x y z)$, as the subgraph of $G$ 
induced by the edges whose interiors lie in the interior of the disk bounded by $x y z$. Note that no edge of $x y z$ lies in int $(x y z)$. We define the exterior of $x y z$, notated ext $(x y z)$, as the subgraph induced by the edges whose interiors lie outside of the disk bounded by $x y z$.

The next lemma is an easy and well-known fact about representativity. The curve it provides will allow us to find a suitable set of edges, whose deletion produces a suitable planar graph.

Lemma 26. If $G$ is a graph embedded in a surface, then there is a closed, non-contractible curve $\alpha$ such that $|\alpha \cap G|=\operatorname{rep}(G)$ and $\alpha \cap G \subseteq V(G)$.

We will need the following well-known theorem of Tutte [11], which, notably, implies that 4-connected planar graphs are Hamiltonian.

Theorem 27 (Tutte). Let $G$ be a plane graph, and let e and $f$ be distinct edges of $G$ such that $e$ is not a cut-edge and such that $e$ and $f$ both lie in some cycle that is contained in the boundary of some face. Then $G$ contains a cycle $C$ that satisfies the following:

(1) $e$ and $f$ are edges of $C$;

(2) Every C-bridge has at most three vertices of attachment;

(3) If a $C$-bridge $B$ shares a vertex with a cycle $C^{\prime}$ such that $C^{\prime}$ contains e and is contained in the boundary of some face, then $B$ has exactly two vertices of attachment.

We will need the following lemma, which comes from [10].

Lemma 28 (Sanders). Let $G$ be a 4-connected plane graph. If $C$ is a cycle of $G$ of length at least four, and $\operatorname{int}(C)$ is 3-connected, then $\operatorname{int}(C)$ has a Hamilton cycle containing any three edges of $C$.

We will also use the following lemma, which is a slight modification of Lemma 2.3 in $[5]$.

Lemma 29 (Ding, Oporowski, Sanders, Vertigan). Let $G$ be a 4-connected plane triangulation with the cycle $x y z$ as the boundary of the infinite face, and suppose that $z$ has degree greater than three. Then $G$ has an edge-partition $\{A, B\}$ such that the following hold:

(1) Each of $G[A], G[B]$ contains $x y z$ and is outerplanar;

(2) Every path in A from $x$ or $y$ to $z$ uses $x z$ or $y z$;

(3) B has no path between any of $x, y, z$ except those contained in xyz;

(4) A contains every edge of $G$ that has an endpoint in $\{x, y\}$. 
Proof. Let $G$ be a 4 -connected plane triangulation with the cycle $x y z$ as the boundary of the infinite face, and suppose that $z$ has degree greater than three. Let $C$ be the cycle of $G$ that bounds the infinite face of $G-z$. Let $v_{x}$ be the unique neighbor of $x$ in $C$ that is not $y$, and let $v_{y}$ be the unique neighbor of $y$ in $C$ that is not $x$. Let $D=\left\{v_{x} x, v_{y} y, x y\right\}$. Since $G$ is 4-connected, we know that $v_{x} \neq v_{y}$. And since $z$ has more than three neighbors in $G$, we know that either $|V(G-z)|=3$ or $|E(C)| \geqslant 4$. Therefore, whether trivially or by Lemma 28, we know that $G-z$ has a Hamiltonian cycle $H$ containing $D$.

Let $X$ consist of the edges of $H$ together with $E(x y z)$ and the edges of $G$ lying in the disk bounded by $H$. Let $Y$ consist of $E(x y z)$ together with the edges of $G$ whose interiors lie outside of the disk bounded by $H$.

Notice that every vertex of $G[A]$ is incident to the face of $G[A]$ that lies outside of the disk bounded by $H$ and inside of the disk bounded by $x y z$; therefore $G[A]$ is outerplanar. Notice that every vertex of $G[B]$ is contained in the face of $G[B]$ that contains the disk bounded by $H$; therefore $G[B]$ is outerplanar. Hence condition (1) of the lemma holds. Since $A$ contains no edges incident to $z$ except for $x z$ and $y z$, we know that every path in $A$ from $x$ or $y$ to $z$ uses $x z$ or $y z$. Hence condition (2) of the lemma holds. Since the only edges in $B$ that have an endpoint in $\{x, y\}$ are $\{x y, x z, y z\}$, we know that $B$ has no path between any of $x, y, z$ except those contained in $x y z$. Hence condition (3) of the lemma holds. And finally, since every edge with an endpoint in $\{x, y\}$ is either in $x y z$, in $H$, or contained in the disk bounded by $H$, we know that $A$ contains all such edges. Hence condition (4) of the lemma holds.

The following theorem is a strengthening of Theorem 2.2 from [5].

Theorem 30. If $G$ is a plane graph and $v$ is a vertex of $G$, then the edges of $G$ can be bipartitioned into $\{S, T\}$ such that $G[S]$ and $G[T]$ are series-parallel and all the edges incident to $v$ lie in $S$.

Proof. Let $\mathcal{S}$ consist of all triples $(G, e, f)$ satisfying the following three conditions:

(1) $G$ is a plane graph;

(2) The edges $e$ and $f$ of $G$ are distinct, incident, and co-facial;

(3) $G$ does not admit the desired edge-partition with respect to $v$, where $v$ is taken to be the vertex shared by $e$ and $f$.

Let $(G, e, f) \in \mathcal{S}$ be a triple such that $G$ has the fewest vertices. Without loss of generality, we may suppose that $G$ is a triangulation, and via a stereographic projection, we may suppose that $e$ and $f$ lie on the infinite face. Clearly $|V(G)|>4$.

Case 1. Suppose that $G$ is 4-connected. Then Theorem 27 yields a Hamiltonian cycle $H$, which contains $e$ and $f$. Let $v$ be the vertex shared by $e$ and $f$. Since $e, f$, and $v$ lie on the boundary of the infinite face, we see that all edges incident to $v$ lie either on $H$ or in the disk bounded by $H$. Let $S$ be the graph induced by $H$ and the edges that lie inside of the disk bounded by $H$. Let $T$ be the graph induced by the edges that lie outside of 
the disk bounded by $H$. Then $S$ and $T$ are outerplanar and therefore series-parallel, and all the edges incident to $v$ lie in $S$.

Case 2. Suppose that $G$ is not 4-connected. Then $G$ has a separating triangle. Let $x y z$ be a separating triangle such that $\operatorname{int}(x y z)$ is minimal with respect to number of vertices. If $e$ and $f$ are contained in $x y z$, then $x y z$ is the boundary of the infinite face, and int $(x y z)$ is not connected; this contradicts the fact that the interior of a separating triangle in a (simple) plane triangulation is connected. Therefore one of $e, f$ is not contained in $x y z$. Furthermore, notice that $x y z$ contains at most one edge on the boundary of the infinite face. Therefore, without loss of generality, we may assume that if $x y z$ contains an edge of the infinite face, then $e=x y$. Let $I=x y z \cup \operatorname{int}(x y z)$, and let $E=x y z \cup \operatorname{ext}(x y z)$. Notice that $e$ and $f$ lie in $E$. By minimality, we know that $E$ has an edge-partition $\left\{S^{\prime}, T^{\prime}\right\}$ into two series-parallel graphs such that the edges incident to $v$ are contained in $S^{\prime}$.

If there is only one vertex $u$ in the interior of $x y z$, then we obtain the desired partition of the edges of $G$ by letting $S=S^{\prime} \cup\{u x, u y\}$ and $T=T^{\prime} \cup u z$. Since the property of being series-parallel is retained under doubling edges and subdividing edges, this partition is as desired.

Suppose, then, that there is more than one vertex in $\operatorname{int}(x y z)$. Since the $\operatorname{int}(x y z)$ is minimal with respect to vertices, we know that $I$ has no separating triangles. Hence $I$ is 4-connected, and the degrees of $x, y$, and $z$ in $I$ are each greater than three. Thus $I$ has an edge-partition $\{A, B\}$ as specified in Lemma 29. Let $S=S^{\prime} \cup(A \backslash\{x y, x z, y z\})$, and let $T=T^{\prime} \cup(B \backslash\{x y, x z, y z\})$. Since $A$ contains all edges of $I$ with an endpoint (with respect to $G[I])$ in $\{x, y\}$, we know that any edges of $G[I]$ that are incident to $v$ lie in $S$. Recall that $A$ contains no paths between $x$ or $y$ and $z$ except $x z$ and $y z$. Therefore we may obtain $S$ from $S^{\prime}$ by doubling and subdividing edges and by adding leaves to $x$ and $y$. Therefore $S$ is series-parallel. Recall also that $B$ contains no paths between any of $x, y, z$ except those contained in $x y z$. Therefore we may obtain $T$ from $T^{\prime}$ by adding leaves to $z$. Therefore $T$ is series-parallel.

\section{The case of the torus - main result}

We now prove our main result on toroidal graphs.

Theorem 31. If $G$ is a toroidal graph, then there is a bipartition $\{X, Y\}$ of $E(G)$ such that $\operatorname{tw}(G / X) \leqslant 3$ and $\operatorname{tw}(G / Y) \leqslant 4$.

Proof. Let $G$ be a toroidal graph. By Lemmas 15 and 14, we may suppose that $G$ is cubic and 2-connected. Our first goal is to find a suitable set $Z$ of edges that satisfies a few specified properties, most notably that $G \backslash Z$ is planar. If there is a closed, noncontractible curve on the torus that meets $G$ at precisely one interior point of one edge $e$, then let $Z=\{e\}$. Otherwise, we must look more closely to find $Z$. Suppose that $G$ admits no such curve on the torus. Then the closure of each face of $G$ is a disk.

By Lemma 26, there exists a closed, homotopically non-trivial curve $\Gamma$ on the torus such that $\Gamma \cap G \subset V(G)$ and $|\Gamma \cap G|=\operatorname{rep}(G)$. We now prove the following: 
Claim 32. No face of $G$ contains more than one connected component of $\Gamma \backslash G$.

Let $F$ be a face of $G$, and assume that $F$ contains two distinct connected components $A$ and $A^{\prime}$ of $\Gamma \backslash G$. Note that for every point $x$ in $\Gamma \cap G$, every open disk about $x$ intersects at least two distinct faces; for otherwise we could very easily find a new curve homotopically equivalent to $\Gamma$ that intersects $G$ one fewer times than does $\Gamma$. Therefore, since $F$ is a disk, we know that $\Gamma \backslash\left(A \cup A^{\prime}\right)$ consists of two connected components $B$ and $B^{\prime}$ and at least one of $B \cup F$ and $B^{\prime} \cup F$ is homotopically non-trivial. Without loss of generality, assume that $B \cup F$ is homotopically non-trivial. Let $\Gamma^{\prime}$ be the curve consisting of $B$ and a segment whose endpoints are the two vertices in $\bar{B} \cap F$ and whose interior lies in the interior of $F$. Then $\Gamma^{\prime}$ is a homotopically non-trivial curve that hits $G$ only at vertices and that hits $G$ fewer times than does $\Gamma$. This contradicts the minimality of $\Gamma$. Therefore Claim 32 holds.

Since every face of $G$ is disk, and since $|\Gamma \cap G|=\operatorname{rep}(G)$, Claim 32 implies that $|\Gamma \cap e| \leqslant 1$, for every $e \in E(G)$.

Let $v_{1}, v_{2}, \ldots, v_{k}$ be the vertices contained in $\Gamma$. The rotation scheme at $v_{i}$, for $i \in$ $\{1, \ldots, k\}$, will be as follows: $e_{i}, \Gamma, e_{i}^{\prime}, e_{i}^{\prime \prime}, \Gamma$, where $e_{i}, e_{i}^{\prime}$, and $e_{i}^{\prime \prime}$ are the three edges incident to $v_{i}$.

We will now alter $\Gamma$ slightly to produce a curve $\Gamma^{\prime}$ that will yield a desirable set of edges; a set whose deletion produces a planar graph. For each $v_{i}$, let $B_{i}$ be an open disk such that the following hold:

(1) $v_{i} \in B_{i}$

(2) $B_{i} \cap G$ is an open star;

(3) $\overline{B_{i} \cap G}$ equals $\overline{B_{i}} \cap G$ and contains none of $V(G)-v_{i}$.

Let $\Gamma^{\prime}$ be the curve obtained from $\Gamma$ by replacing, for every $i \in\{1, \ldots, k\}$, the path $\overline{B_{i}} \cap \Gamma$ with the path on the boundary of $B_{i}$ that intersects $e_{i}$. Then $\Gamma^{\prime} \cap G$ consists of precisely one interior point from $e_{i}$, for each $i \in\{1, \ldots, k\}$. Let $Z=\left\{e_{1}, \ldots, e_{k}\right\}$. Note that $\left|\Gamma^{\prime} \cap G\right|=\operatorname{rep}(G)$. We now prove the following:

Claim 33. The edges of $Z$ are pairwise non-adjacent.

Assume, en route to a contradiction, that $e_{i}$ and $e_{j}$ are adjacent at vertex $v$, with $i \neq j$. Then by Claim 32 and the fact that $\left|\Gamma^{\prime} \cap e_{i}\right|=\left|\Gamma^{\prime} \cap e_{j}\right|=1$, it follows that $|i-j|=1$. Let $B$ be an open disk such that the following hold:

(1) $v \in B$

(2) $\Gamma^{\prime} \cap G$ is an open star;

(3) $\overline{B \cap G}$ equals $\bar{B} \cap G$ and contains none of $V(G)-v$.

Let $\Gamma^{\prime \prime}$ be the curve obtained from $\Gamma^{\prime}$ by replacing the path $\Gamma^{\prime} \cap B$ with the path on the boundary of $B$ that intersects neither $e_{i}$ nor $e_{j}$. Then $\Gamma^{\prime \prime}$ is a non-contractible curve and $\left|\Gamma^{\prime \prime} \cap G\right|<\left|\Gamma^{\prime} \cap G\right|$. This contradicts the minimality of $\left|\Gamma^{\prime} \cap G\right|$. Hence Claim 33 holds. 


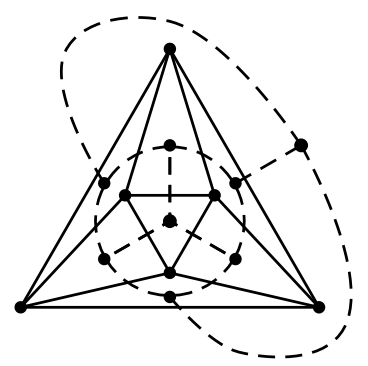

$M_{6}$ and $M_{6}^{*}$

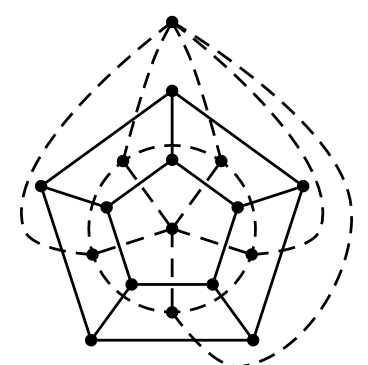

$M_{10}$ and $M_{10}^{*}$

Figure 11: $M_{6}, M_{10}$, and their plane duals.

Therefore $G \backslash Z$ is planar and the face created by the deletion of $Z$ is bounded by two cycles $S$ and $T$. Let $G^{\prime}$ be $G \backslash Z$ with the vertices of degree two suppressed, and embed $G^{\prime}$ in the plane such that $S$ and $T$ are facial cycles. Let $H$ be a plane dual of $G^{\prime}$, and let $s$ and $t$ be the vertices of $H$ associated with the faces $S$ and $T$ of $G^{\prime}$. Since $G^{\prime}$ is cubic, we know that $H$ is a triangulation.

Using Theorem 30, we bipartition $E(H)$ into $E_{1}^{\prime}$ and $E_{2}^{\prime}$ such that all the edges incident to $s$ lie in $E_{1}^{\prime}$, and $H \backslash E_{1}^{\prime}$ and $H \backslash E_{2}^{\prime}$ are series-parallel. Let $E_{t}$ consist of the edges incident to $t$, and let $E_{1}=E_{1}^{\prime} \backslash E_{t}$ and $E_{2}=E_{2}^{\prime} \cup E_{t}$. Then $H \backslash E_{2}$ is a subgraph of $H \backslash E_{2}^{\prime}$ and therefore is series-parallel. Hence, by Lemma 21, we know that $G / E_{2}$ is series-parallel.

We see that $H \backslash E_{1}$ can be obtained from $H \backslash E_{1}^{\prime}$ by adding a vertex to $V\left(H \backslash E_{1}^{\prime}\right)$ and adding any number of edges between that vertex and the rest of the vertices in $H \backslash E_{1}^{\prime}$. We know that the unique plane dual of $M_{6}$ is the cube, and the unique plane dual of $M_{10}$ is the double 5-wheel. See Figure 11.

Furthermore, both the cube minus any vertex and the double 5-wheel minus any vertex contain a $K_{4}$-minor. Therefore, since $H \backslash E_{1}^{\prime}$ contains no $K_{4}$-minor, we know that $H \backslash E_{1}$ contains no minor isomorphic to the cube or the double 5-wheel. Hence, by Lemma 21, we know that $G^{\prime} / E_{1}$ has no minor isomorphic to $M_{6}$ or $M_{10}$. And by planarity, we know that $G^{\prime} / E_{1}$ has no minor isomorphic to $K_{5}$ or $M_{8}$. Hence $\operatorname{tw}\left(G^{\prime} / E_{2}\right) \leqslant 2$ and $\operatorname{tw}\left(G^{\prime} / E_{1}\right) \leqslant 3$. Furthermore, $S \subseteq E_{1}$ and $T \subseteq E_{2}$.

Let $u$ be the vertex to which $S$ is contracted in $G^{\prime} / E_{1}$, and let $v$ be the vertex to which $T$ is contracted in $G^{\prime} / E_{2}$. Then we can construct a tree decomposition of $G /\left(E_{1} \cup Z\right)$, with width at most four, by adding $u$ to every bag in the tree decomposition of $G^{\prime} / E_{1}$. And we can construct a tree decomposition of $G / E_{2}$, with width at most three, by adding $v$ to every bag in the tree decomposition of $G^{\prime} / E_{2}$. Let $X=E_{1} \cup Z$ and $Y=E_{2}$. Then $\{X, Y\}$ is the desired bipartition of $E(G)$. 


\section{Acknowledgement}

We are grateful to an anonymous referee, who pointed out a few errors in an earlier version of this paper, and suggested using piecewise-linear embeddings to simplify some topological arguments.

\section{References}

[1] Stefan Arnborg, Andrzej Proskurowski, and Derek G. Corneil. Forbidden minors characterization of partial 3-trees. Discrete Math., 80(1):1-19, 1990.

[2] Gary Chartrand, Dennis Geller, and Stephen Hedetniemi. Graphs with forbidden subgraphs. J. Combin. Theory Ser. B, 10:12-41, 1971.

[3] Erik D. Demaine, Mohammad TaghiHajiaghayi, and Bojan Mohar. Approximation algorithms via contraction decomposition. Combinatorica, 30(5):533-552, 2010.

[4] Reinhard Diestel. Graph theory, volume 173 of Graduate Texts in Mathematics. Springer, Heidelberg, fourth edition, 2010.

[5] Guoli Ding, Bogdan Oporowski, Daniel P. Sanders, and Dirk Vertigan. Surfaces, tree-width, clique-minors, and partitions. J. Combin. Theory Ser. B, 79(2):221-246, 2000 .

[6] Daniel Gonçalves. Edge partition of planar graphs into two outerplanar graphs. In STOC'05: Proceedings of the 37th Annual ACM Symposium on Theory of Computing, pages 504-512. ACM, New York, 2005.

[7] Kiran S. Kedlaya. Outerplanar partitions of planar graphs. J. Combin. Theory Ser. $B, 67(2): 238-248,1996$.

[8] C. St. J. A. Nash-Williams. Edge-disjoint spanning trees of finite graphs. J. London Math. Soc., 36:445-450, 1961.

[9] James Oxley. Private communication, 2002.

[10] Daniel P. Sanders. On Hamilton cycles in certain planar graphs. J. Graph Theory, 21(1):43-50, 1996.

[11] W. T. Tutte. A theorem on planar graphs. Trans. Amer. Math. Soc., 82:99-116, 1956.

[12] W. T. Tutte. On the problem of decomposing a graph into $n$ connected factors. J. London Math. Soc., 36:221-230, 1961. 